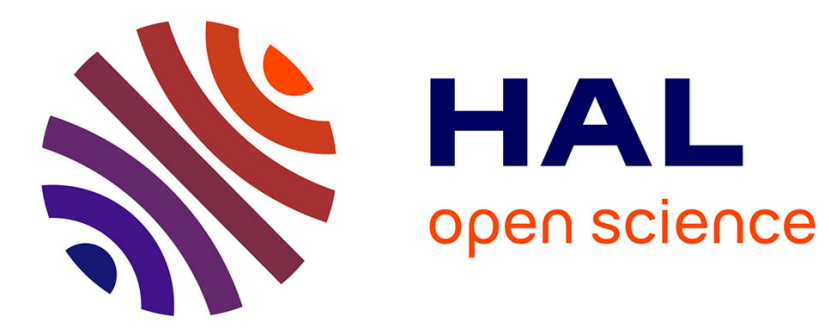

\title{
An inverse approach to determine the non-linear properties of induction heat-treated steels
}

André Niclas, Lament Bourgeois

\section{To cite this version:}

André Niclas, Lament Bourgeois. An inverse approach to determine the non-linear properties of induction heat-treated steels. European Journal of Mechanics - A/Solids, 2000, 19, pp.69-88. 10.1016/S0997-7538(00)00152-2 . hal-00111286

\section{HAL Id: hal-00111286 \\ https://hal.science/hal-00111286}

Submitted on 2 Mar 2019

HAL is a multi-disciplinary open access archive for the deposit and dissemination of scientific research documents, whether they are published or not. The documents may come from teaching and research institutions in France or abroad, or from public or private research centers.
L'archive ouverte pluridisciplinaire HAL, est destinée au dépôt et à la diffusion de documents scientifiques de niveau recherche, publiés ou non, émanant des établissements d'enseignement et de recherche français ou étrangers, des laboratoires publics ou privés. 


\title{
An inverse approach to determine the non-linear properties of induction heat-treated steels
}

\author{
André Niclas ${ }^{a}$, Lament Bourgeois ${ }^{b}$ \\ ${ }^{a}$ Laboratoire de Microstructure et Mécanique des Matériaux, Ecole Nationale Supérieure des Arts et Métiers, 151 Boulevard de \\ l'Hopital, 75013 Paris, France \\ ${ }^{\mathrm{b}}$ Laboratoire de Mécanique des Solides, Ecole Polytechnique, 91128 Palaiseau Cedex, France
}

\begin{abstract}
The identification of the through-the-thickness variation of the non-linear properties of a steel structure due to a heat treatment by induction is considered. An inverse approach to that problem is proposed, which is based on a 4-point bending test of a heat-treated parallelepipedic bar. It includes a layer-removal method for measuring residual stresses. Precisely, two different inverse methods are described. For a medium carbon steel, an application based on experimental data is performed, which shows the efficiency of our approach and a good agreement between the two methods.
\end{abstract}

inverse problem / plasticity / X-ray diffraction / heat treatment / residual stresses

\section{Introduction}

The optimization of mass, volume and strength among others, constrains the engineer to design steel components with local reinforcement resulting from specific treatments. Superficial treatments such as induction hardening, shot-peening and nitriding are often practiced. These processes introduce residual stresses and gradients in mechanical properties within the material, precisely, gradients in plastic properties (for example the tensile yield strength and the strain hardening modulus). Various computational tools provide the designer with accurate residual stress predictions within the component. However, the gradient in the plastic properties is unknown and has to be identified.

In the framework of classical tensile tests, establishing the relationship between microstructural composition and plastic properties would necessitate the realization of homogeneous test bars composed of different types of microstructure. These tests would give uncertain results, first because homogeneous bars are very difficult to produce and, second, because of brittleness (test bars could fail before the tensile yield strength is reached).

This paper is concerned with a new methodology to determine the plastic constitutive parameters of heat treated material, with application to medium carbon steel. It is based on the analysis of a bending test by inverse methods. We first describe the heat treament and its consequences and, secondly, the bending test. Then, following a brief presentation of X-ray diffraction, which is used to calculate the stress fields in our test bars, two different inverse approaches are proposed based on the bending test to determine the gradient in plastic properties within the affected layer. The first one is based on measurements of the stress fields before and after the test. The second one consists of minimizing the discrepancy between the data obtained during the real test and those provided by a simulation of the test. 


\section{The heat treatment}

The treatment which is studied here consists of a high-frequency induction hardening. The industry currently uses this process, which changes the microstructural composition within a layer of adjustable thickness. Its physical principles are summarized as follows (see for example (Davies and Simpson, 1979) for details).

(i) A high frequency current passes through an inductor and creates a magnetic field.

(ii) Eddy currents are principally induced on the surface of the piece placed near the field coil.

(iii) These currents heat the piece, locally raising the temperature above the austenitization threshold (AC1 point). A transformation into austenite takes place within a depth of several millimetres.

(iv) The specimen is finally quenched, resulting in austenite being transformed into martensite (the hardest phase of steel).

The mechanical state after treatment is characterized by the presence of a residual stress field and a gradient in microstructural properties. Residual stresses have been created by variations in temperature and phase changes during treatment, which are both responsible for volume changes. Since the thermal and metallurgic evolutions at the surface and within the component are very different, internal strains develop to balance these volume variations. At the end of the treatment, the residual stress field can be measured by many destructive methods (removal of matter and X-ray diffraction, hole drilling, etc.).

Metallurgical transformations, which consist of a modification of the crystallographic structure, also occur during treatment: the arrangements of the atoms in grains and grain shapes change and generate many phases which have different microscopic aspects and different mechanical properties. The kinetics of thermal phenomena have a direct influence on the metallurgical transformation, which is described in TTT and CCT diagrams (see for example (Barralis and Maeder, 1983) for definitions) that are specific to each metal. The industrial processes such as induction hardening are intended to transform the initial material into a martensitic phase within a desired depth of the component. This transformation necessitates an increase in temperature above the $\mathrm{AC} 1$ point (often higher than $750^{\circ} \mathrm{C}$ ) to obtain austenitic phase, followed by a very fast quench (the quenching rate must be higher than $50^{\circ} \mathrm{C} \cdot \mathrm{s}^{-1}$ ). The majority of austenite is then transformed into martensite with improved mechanical properties. A gradient in the metallurgical composition is often expected within the component because the metal has not been totally austenitized during heating or because the cooling rate has been insufficient to obtain $100 \%$ martensite. At the macroscopic scale, this phenomenon results in gradients in mechanical properties.

We postulate that the elastic properties of the metal are not affected by the heat treatment. This assumption lies in the fact that the crystalline structure of the different phases (martensite, ferrite and other) is the same (B.C.C. structure). Only the plastic properties may have been altered by the heat treatment.

\section{The 4-point bending test}

This section is dedicated to the description and the modelling of the 4-point bending test.

\subsection{The objective}

This paper addresses the identification of the tensile yield strength and the strain hardening modulus, which are assumed to fully characterize the plastic response of the material within the layer affected by heat treatment. This identification can only be done by introducing additional irreversible strain in this layer through a mechanical test, here a 4-point bending test, which has at least three advantages (see figure 1 for notation). 


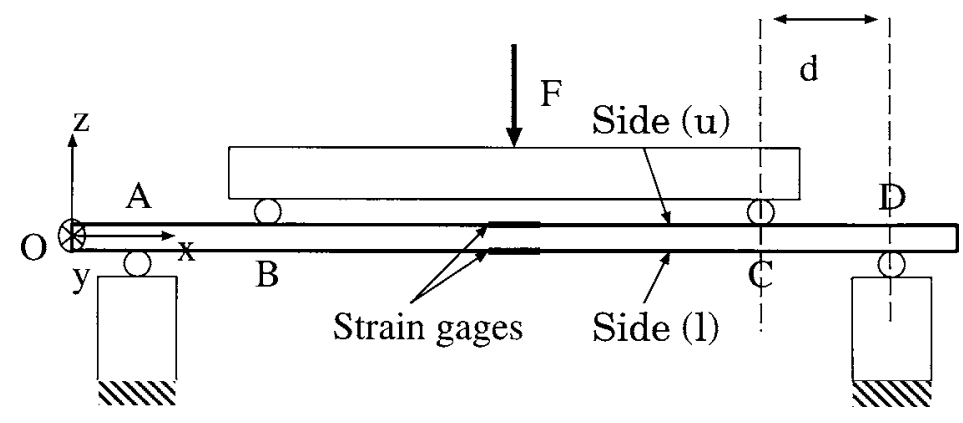

Figure 1. The 4-point bending test.

(i) It is easy to perform.

(ii) The applied bending moment is uniform in directions $x$ and $y$, which motivates the assumption that total strains, plastic strains and stresses are only depending on $z$.

(iii) During such a test, the outer layers are the most stressed (tensile stresses are created on one side, called side $(l)$, compression stresses on the other side, called side $(u)$ ). It is an advantage here because the outer layers are precisely those in which the tensile yield strength are the largest.

\subsection{The mechanical fields}

The test bar is a parallelepided which occupies the domain $\Omega=\left[0, L_{1}\right] \times\left[0, L_{2}\right] \times[-h, h]$ in the Cartesian coordinates $(x, y, z), 2 h$ being much smaller than $L_{1}$ and $L_{2}$. To simplify notations, variables $(x, y, z)$ will often be designated by $(1,2,3)$.

We assume that before and during the bending test, total strain, plastic strain and stress fields only depend on $z$. This assumption, partly motivated earlier, relies on the fact that:

(i) the heat treatment is uniform in directions $x$ and $y$,

(ii) the mechanical fields generated during bending between the two points $B$ and $C$ are also independent of $x$ and $y$, provided we consider a region which is a not too close to these two points (according to Saint-Venant's principle).

Denote by $\boldsymbol{u}, \boldsymbol{\varepsilon}, \boldsymbol{\varepsilon}^{\mathrm{p}}$ and $\boldsymbol{\sigma}$ displacement, total strain, plastic strain and stress, respectively.

Since $\sigma_{z z}(x, y, \pm h)=0$, equilibrium implies $\sigma_{z z}=0$ in $\Omega$. Furthermore, we disregard shear stress

$$
\sigma_{1 z}=\sigma_{2 z}=0 .
$$

The assumed form for the stress field is therefore

$$
\boldsymbol{\sigma}=\left(\begin{array}{ccc}
\sigma_{11}(z) & \sigma_{12}(z) & 0 \\
\sigma_{12}(z) & \sigma_{22}(z) & 0 \\
0 & 0 & 0
\end{array}\right)
$$

The total strain tensor being a function of $z$, the only compatibility equations are

$$
\frac{\partial^{2} \varepsilon_{i j}}{\partial z^{2}}=0 \quad(i, j=1,2),
$$


which are satisfied by

$$
\varepsilon_{i j}=\beta_{i j}-z \gamma_{i j}
$$

where the second-order tensors $\boldsymbol{\beta}$ and $\boldsymbol{\gamma}$ are constant. The tensor $\boldsymbol{\beta}$ defines the strain in the plane of equation $z=0$. We also assume that

$$
\varepsilon_{1 z}=\varepsilon_{2 z}=0 .
$$

We now use the strain-displacement relationship to relate the tensor $\boldsymbol{\gamma}$ introduced in (3) to the displacements. Let $u_{i}(x, y, z)(i=1,2)$ and $w(x, y, z)$ be the components of displacement in directions $x, y$ and $z$. Using (3) and (4), one finds that

$$
\gamma_{i j}=\frac{\partial^{2} w}{\partial x_{i} \partial x_{j}},
$$

revealing that $\partial^{2} w / \partial x_{i} \partial x_{j}$ is constant and that $\boldsymbol{\gamma}$ is nothing but the curvature tensor of the plane of equation $z=0$.

Plastic strain, which satisfies $\operatorname{tr} \boldsymbol{\varepsilon}^{\mathrm{p}}=0$, is assumed to have the expression

$$
\boldsymbol{\varepsilon}^{\mathrm{p}}=\left(\begin{array}{ccc}
\alpha_{11}(z) & \alpha_{12}(z) & 0 \\
\alpha_{12}(z) & \alpha_{22}(z) & 0 \\
0 & 0 & -\alpha_{11}(z)-\alpha_{22}(z)
\end{array}\right) \text {. }
$$

\subsection{Description of the testing device}

The testing device is presented in figure 1. The four contact points transmit, in the region of the test bar situated between points $B$ and $C$, a moment per unit of length (in direction $y$ ) $M_{11}$ which is uniform in directions $x$ and $y$ and of value

$$
M_{11}=-\frac{1}{2} \frac{F d}{L_{2}}=-M
$$

This loading is applied with the following kinematical conditions on curvature:

$$
\gamma_{12}=0, \quad \gamma_{22}=0
$$

Note that, since contact at $B$ and $C$ is not perfect, the moment is actually uniform only at a certain distance away from the two points.

A classical device provides the intensity of the force $F$ and the displacement of its point of application. Furthermore, strain gauges situated on both sides of the test bar provide the strain at the boundary $\varepsilon_{i j}(h)$ and $\varepsilon_{i j}(-h)$. These data yield estimates of the mid-plane strain $\beta_{i j}$ and the curvature $\gamma_{i j}$, by solving the system of equations

$$
\left\{\begin{array}{l}
\varepsilon_{i j}(h)=\beta_{i j}-h \gamma_{i j}, \\
\varepsilon_{i j}(-h)=\beta_{i j}+h \gamma_{i j},
\end{array}\right.
$$

and then estimates of the strain field.

During the test, the load $F$ is first increased from 0 to $F_{m}$, then decreased back to 0 . 


\subsection{The constitutive law}

The zero strain state for the total strain and plastic strain corresponds to the state of the test bar just after the heat treatment. This state will be designated as the "initial state". It is characterized by a residual stress field denoted by $\sigma^{0}$ which is self-equilibrated. We assume that the constitutive law is given by the classical Prager's model (see for example (François et al., 1992)), which corresponds to the following free energy:

$$
W\left(\varepsilon, \varepsilon^{\mathrm{p}}\right)=\sigma^{0}:\left(\varepsilon-\varepsilon^{\mathrm{p}}\right)+\frac{1}{2}\left(\varepsilon-\varepsilon^{\mathrm{p}}\right): C:\left(\varepsilon-\varepsilon^{\mathrm{p}}\right)+\frac{1}{2} H \boldsymbol{\varepsilon}^{\mathrm{p}}: \varepsilon^{\mathrm{p}},
$$

where $\boldsymbol{C}$ is the linear elasticity Hooke's tensor and $H$ is the modulus of strain hardening. We deduce the thermodynamical forces associated with the state variables $\boldsymbol{\varepsilon}$ and $\boldsymbol{\varepsilon}^{\mathrm{p}}$ respectively using

$$
\boldsymbol{\sigma}=\frac{\partial W}{\partial \boldsymbol{\varepsilon}}, \quad \boldsymbol{A}=-\frac{\partial W}{\partial \boldsymbol{\varepsilon}^{\mathrm{p}}},
$$

resulting, more explicitely, in the Hooke's law

$$
\sigma=\sigma^{0}+C:\left(\varepsilon-\varepsilon^{\mathrm{p}}\right)
$$

and

$$
\boldsymbol{A}=\boldsymbol{\sigma}-H \boldsymbol{\varepsilon}^{\mathrm{p}} .
$$

Given the particular form of mechanical fields $\varepsilon, \varepsilon^{\mathrm{p}}$ and $\sigma$ defined in the previous paragraph, equation (7) is rewritten as

$$
\left\{\begin{array}{l}
\sigma_{11}=\sigma_{11}^{0}+\frac{E}{1-v^{2}}\left\{\left(\varepsilon_{11}+v \varepsilon_{22}\right)-\left(\alpha_{11}+v \alpha_{22}\right)\right\}, \\
\sigma_{22}=\sigma_{22}^{0}+\frac{E}{1-v^{2}}\left\{\left(v \varepsilon_{11}+\varepsilon_{22}\right)-\left(v \alpha_{11}+\alpha_{22}\right)\right\}, \\
\sigma_{12}=\sigma_{12}^{0}+\frac{E}{1+v}\left(\varepsilon_{12}-\alpha_{12}\right),
\end{array}\right.
$$

in which $E$ and $v$ are Young's modulus and Poisson's ratio, respectively.

The yield function which defines the Von Mises convex of plasticity is

$$
\mathcal{F}(\boldsymbol{A})=\sqrt{Q(\boldsymbol{A})}-k, \quad Q(\boldsymbol{A})=\frac{3}{2} \boldsymbol{A}^{\prime}: \boldsymbol{A}^{\prime}
$$

where $\boldsymbol{A}^{\prime}$ is the deviatoric part of $\boldsymbol{A}$ and $k$ is the tensile yield strength. The loading and unloading conditions with the classical normality flow rule are

$$
\left\{\begin{array}{l}
\mathcal{F}(\boldsymbol{A}) \leqslant 0, \\
\mathcal{F}(\boldsymbol{A})<0 \rightarrow \dot{\boldsymbol{\varepsilon}}^{\mathrm{p}}=0, \\
\mathcal{F}(\boldsymbol{A})=0 \rightarrow \dot{\boldsymbol{\varepsilon}}^{\mathrm{p}}=\dot{\omega} \boldsymbol{A}^{\prime}, \quad \dot{\omega} \geqslant 0 .
\end{array}\right.
$$

The plastic behaviour of the material is thus characterized solely by the tensile yield strength $k$ and the strain hardening modulus $H$.

To shorten notations, we introduce the following scalars

$$
E^{\prime}=\frac{E}{1-v^{2}}, \quad E^{\prime \prime}=\frac{E}{1+v}, \quad E_{1}=E_{2}=E^{\prime}, \quad E_{3}=E^{\prime \prime},
$$


and simplified indices notation:

$$
\left\{\begin{array} { l } 
{ \sigma _ { 1 } = \sigma _ { 1 1 } , } \\
{ \sigma _ { 2 } = \sigma _ { 2 2 } , } \\
{ \sigma _ { 3 } = \sigma _ { 1 2 } , }
\end{array} \quad \left\{\begin{array}{l}
\varepsilon_{1}=\varepsilon_{11}+v \varepsilon_{22}, \\
\varepsilon_{2}=v \varepsilon_{11}+\varepsilon_{22}, \\
\varepsilon_{3}=\varepsilon_{12} .
\end{array}\right.\right.
$$

For $i=1,2,3$, we define $\beta_{i}, \gamma_{i}$ and $\alpha_{i}$ from the components of tensors $\boldsymbol{\beta}, \boldsymbol{\gamma}$ and $\boldsymbol{\varepsilon}^{\mathrm{p}}$, respectively, exactly as the $\varepsilon_{i}$ 's are defined from the components of tensor $\boldsymbol{\varepsilon}$ just above.

With this notation, relations (3) and (9) simply become

$$
\varepsilon_{i}=\beta_{i}-z \gamma_{i} \quad(i=1,2,3)
$$

and

$$
\sigma_{i}=\sigma_{i}^{0}+E_{i}\left(\varepsilon_{i}-\alpha_{i}\right) \quad(i=1,2,3) .
$$

The initial stress field $\sigma^{0}$ is assumed to depend only on $z$ because heat treatment is uniform in directions $x$ and $y$, as already mentioned. For the same reason, plastic parameters $k$ and $H$ are also assumed to depend only on $z$. Furthermore, the heat treatment being symmetrically applied on both faces of the test bar, one assumes that $k, H$ and $\sigma^{0}$ are symmetrical with respect to the plane of equation $z=0$.

\subsection{Simulation of the bending test}

To solve an inverse problem with the data obtained during the test, it is necessary to propose a modelling of this test (forward calculation). Total strain, plastic strain and stress fields are assumed to have the expressions previously indicated.

Generalized forces are defined by

$$
N_{i}=\int_{-h}^{h} \sigma_{i} \mathrm{~d} z, \quad M_{i}=\int_{-h}^{h} z \sigma_{i} \mathrm{~d} z \quad(i=1,2,3) .
$$

The initial stress field $\sigma^{0}$ is self-equilibrated and, by using (11), the generalized forces are written as

$$
\left\{\begin{array}{l}
N_{i}=E_{i} S\left(\beta_{i}-\left\langle\alpha_{i}\right\rangle\right) \quad(i=1,2,3), \\
M_{i}=-E_{i} I \gamma_{i}-E_{i} S\left\langle z \alpha_{i}\right\rangle \quad(i=1,2,3),
\end{array}\right.
$$

in which we define

$$
S=2 h, \quad I=\frac{2}{3} h^{3}, \quad\langle\cdot\rangle=\frac{1}{2 h} \int_{-h}^{h} \cdot \mathrm{d} z .
$$

The kinematic and static conditions for the forward elastic-plastic calculation are respectively

$$
\gamma_{2}=0, \quad \gamma_{3}=0
$$

and

$$
M_{1}=-M, \quad N_{i}=0 \quad(i=1,2,3) .
$$

The incremental algorithm of the forward calculation is classical (see for example (Nguyen, 1977)); we just mention its two key steps. 
(i) First step: compute the increment of total strain $\Delta \varepsilon$ and the increment of stress $\Delta \sigma$ corresponding to the rate of loading $\Delta M$, the increment of plastic strain $\Delta \varepsilon^{\mathrm{p}}$ being kept constant.

The expression of the rate of total strain is, according to kinematic and static conditions,

$$
\left\{\begin{array}{l}
\Delta \gamma_{2}=0, \quad \Delta \gamma_{3}=0, \\
\Delta \beta_{i}=\left\langle\Delta \alpha_{i}\right\rangle \quad(i=1,2,3), \\
\Delta \gamma_{1}=\frac{1}{E^{\prime} I} \Delta M-\frac{S}{I}\left\langle z \Delta \alpha_{1}\right\rangle .
\end{array}\right.
$$

(ii) Second step: compute the increment of plastic strain $\Delta \varepsilon^{\mathrm{p}}$ such that the new thermodynamical force $\boldsymbol{A}+\boldsymbol{\Delta} \boldsymbol{A}$ remains in the convex of plasticity. We thus impose the consistency condition

$$
\mathcal{F}(\boldsymbol{A}+\boldsymbol{\Delta} \boldsymbol{A})=0 .
$$

According to the flow rule, we furthermore impose that $\Delta \boldsymbol{\varepsilon}^{\mathrm{p}}$ is proportional to $\boldsymbol{A}^{\prime}+\boldsymbol{\Delta} \boldsymbol{A}^{\prime}$. We deduce

$$
\Delta \varepsilon^{\mathrm{p}}=\Omega\left(\boldsymbol{A}^{\prime}+2 \mu \Delta \boldsymbol{\varepsilon}^{\prime}\right)
$$

$\mu$ being the shear modulus, $\boldsymbol{\varepsilon}^{\prime}$ being the deviatoric part of $\boldsymbol{\varepsilon}$, and $\Omega$ being defined by

$$
\Omega=\frac{1}{2 \mu+H} \frac{Q(\boldsymbol{A}+2 \mu \boldsymbol{\Delta} \boldsymbol{\varepsilon})-k}{Q(\boldsymbol{A}+2 \mu \boldsymbol{\Delta} \boldsymbol{\varepsilon})} .
$$

Since the total strain, plastic strain and stress fields depend only on $z$, the forward elastic-plastic computation needs only a discretization in the thickness direction. Subsequently, the mechanical fields are considered as $m$ vectors ( $m$ : number of layers used in the thickness direction). The discretized parameters governing plastic behaviour $(k, H)$ are also $m$-vectors.

We point out that the component $\sigma_{12}^{0}$ of the initial stress field $\sigma^{0}$ is very close to 0 in comparison with the other components; this is due to the heat treatment procedure and inferred from X-ray diffraction measurements, discussed in the next section. Kinematic and static conditions for the 4-point bending test are such that the same conclusion applies to the component $\sigma_{12}$ at any step of the bending test.

\section{Determination of the residual stress field}

To apply our methodology, we need to measure the residual stress field within the treated test bars. It is determined by using X-ray diffraction. This method, detailed in (Lebrun, 1996), is based on the measurement of the interreticular distance between the crystallographic planes. The acquisition of the stress field within the piece necessitates the removal of the layers of material and successive diffraction measurements. Matter removal is accomplished by an electropolishing method, which does not perturbe the stress, to the contrary of, e.g., mechanical polishing. But the change in geometry induces a redistribution of the initial stress, which has to be recalculated from the successive diffraction measurements. Some reconstruction formulae for simple geometries are proposed in (Moore and Evans, 1956), their extension to any geometry being solved in (Ballard and Constantinescu, 1994). For our particular geometry (stress field of form (2)) and if layers of constant thickness are removed from the whole surface, the following Moore and Evans formulae provide the stress field $\sigma$ before matter removal as a function of stresses $\sigma^{\mathrm{m}}$ measured on the successive surfaces $(S=2 h$ being 


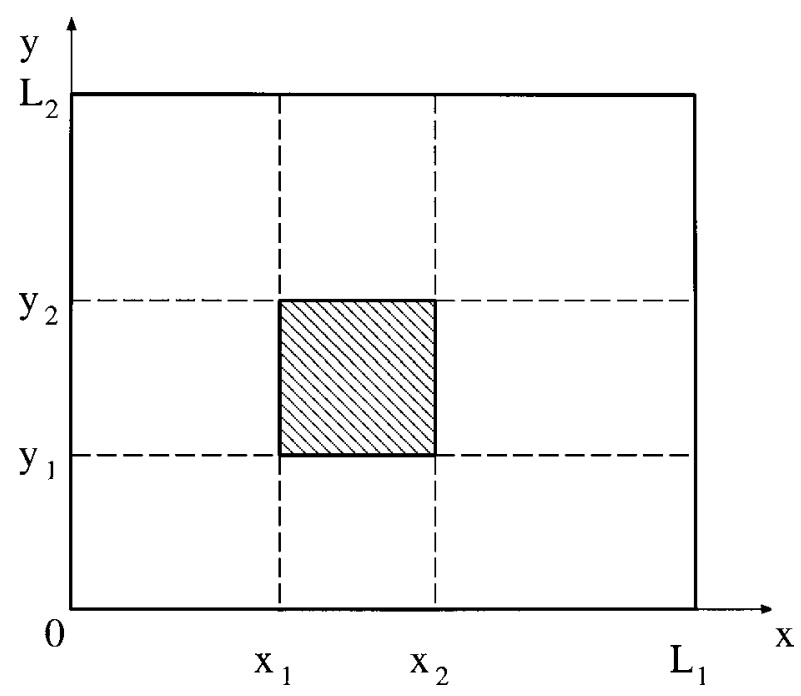

Figure 2. Partial removal of matter.

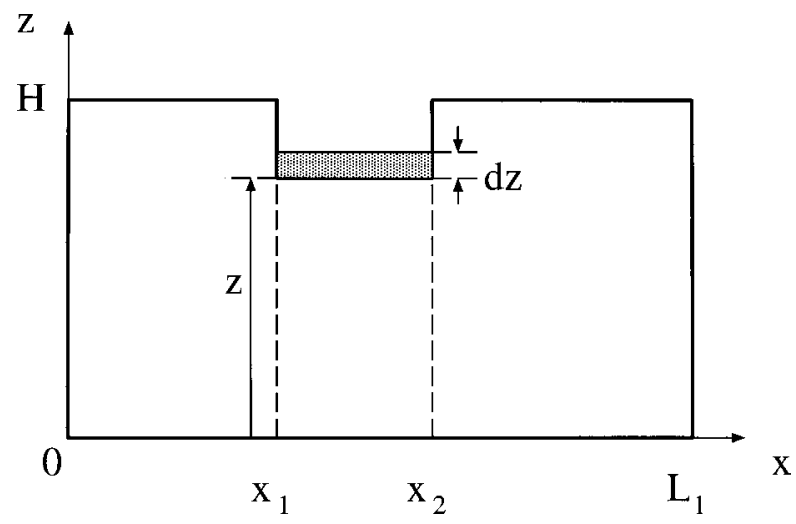

Figure 3. Partial removal of matter.

the total width of the piece):

$$
\sigma_{i}\left(z_{1}\right)=\sigma_{i}^{\mathrm{m}}\left(z_{1}\right)+2 \int_{z_{1}}^{S} \frac{\sigma_{i}^{\mathrm{m}}(z)}{z} \mathrm{~d} z-6 z_{1} \int_{z_{1}}^{S} \frac{\sigma_{i}^{\mathrm{m}}(z)}{z^{2}} \mathrm{~d} z \quad(i=1,2,3) .
$$

For practical reasons, only a part of the surface has been removed in the framework of our study, precisely a square defined by $\left[x_{1}, x_{2}\right] \times\left[y_{1}, y_{2}\right]$ (see figures 2 and 3 ). In this configuration, the reconstruction formulae, determined in (Bourgeois, 1998), become:

$$
\sigma_{i}\left(z_{1}\right)=\sigma_{i}^{\mathrm{m}}\left(z_{1}\right)+\int_{z_{1}}^{S} f_{1}(z) \sigma_{i}^{\mathrm{m}}(z) \mathrm{d} z-z_{1} \int_{z_{1}}^{S} g_{1}(z) \sigma_{i}^{\mathrm{m}}(z) \mathrm{d} z \quad(i=1,3)
$$

and

$$
\sigma_{i}\left(z_{1}\right)=\sigma_{i}^{\mathrm{m}}\left(z_{1}\right)+\int_{z_{1}}^{S} f_{2}(z) \sigma_{i}^{\mathrm{m}}(z) \mathrm{d} z-z_{1} \int_{z_{1}}^{S} g_{2}(z) \sigma_{i}^{\mathrm{m}}(z) \mathrm{d} z \quad(i=2,3),
$$

where $r_{1}=L_{1} /\left(y_{2}-y_{1}\right)$ and $r_{2}=L_{2} /\left(x_{2}-x_{1}\right)$. The functions $f_{1}$ and $g_{1}$ introduced in (13) and (14) are

$$
\begin{aligned}
& f_{1}(z)=\frac{6 z\left(S^{2}\left(r_{1}-1\right)+z^{2}\right)-4\left(S^{3}\left(r_{1}-1\right)+z^{3}\right)}{4\left(S^{3}\left(r_{1}-1\right)+z^{3}\right)\left(S\left(r_{1}-1\right)+z\right)-3\left(S^{2}\left(r_{1}-1\right)+z^{2}\right)^{2}}, \\
& g_{1}(z)=\frac{12 z\left(S\left(r_{1}-1\right)+z\right)-6\left(S^{2}\left(r_{1}-1\right)+z^{2}\right)}{4\left(S^{3}\left(r_{1}-1\right)+z^{3}\right)\left(S\left(r_{1}-1\right)+z\right)-3\left(S^{2}\left(r_{1}-1\right)+z^{2}\right)^{2}} .
\end{aligned}
$$

The functions $f_{2}$ and $g_{2}$ have the same expressions as $f_{1}$ and $g_{1}$ with $r_{1}$ replaced by $r_{2}$. Setting $r_{1}$ and $r_{2}$ to 1 in (15), which corresponds to a removal of matter on the whole surface, (13)-(14) degenerate into the classical Moore and Evans' formulae. One must bear in mind that the formulas (13)-(14) are valid only for sufficiently small depths, say up to one fourth of the total width. Beyond that, geometry is too altered, so that the assumptions used to establish them are not valid.

Apart from the determination of the stress field, X-ray diffraction is also useful in determining the microstructural state within the piece at the end of treatment. Metallographic microsections give qualitative 


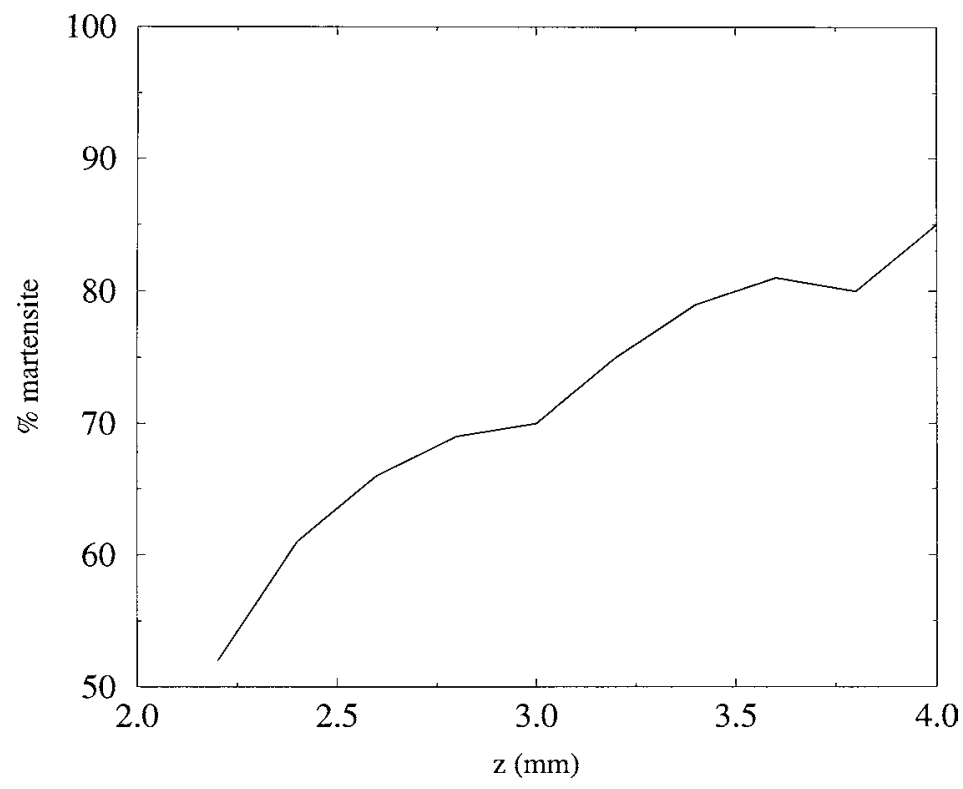

Figure 4. Proportion of martensite as a function of depth.

indications, microhardness measurements and analyses of X-ray diffraction peaks provide quantitative information. For a two-phase metal, the diffraction peak is the sum of the diffraction peak of each phase. It is possible to separate these two peaks. Since the metallurgical proportion and the relative peak area of one phase are proportional, X-ray diffraction provides the composition of the treated metal at a given point (see (Lebrun, 1996) for details). Figure 4 shows the variation of the metallurgical composition with depth for a particular test bar. The treated zone is a mixture of martensitic and ferritic phases. The proportion of martensite decreases with depth. These considerations are confirmed by microhardness measurements and observations of the microstructure. Figure 5 shows the variation of Vickers microhardness with depth. It has to be compared to the previous variation of the proportion of martensite. Figures 6 and 8 respectively show the ferrito-perlitic and martensitic microstructures observed, figure 7 shows the mixed zone between these two microstructures. In fact, the martensitic phase is not pure but has a small proportion of ferrite which has not been austenitized during heat treatment.

In what follows, some of our assumptions will by justified thanks to these observations.

\section{Identification of the plasticity model}

We are now in a position to consider the main objective of this paper: the determination of the tensile yield strength $k$ and the strain hardening modulus $H$ for a steel test bar which was submitted to a heat treatment. These two parameters are function of coordinate $z$. Two different methods are considered to solve the problem.

(i) The first one is based on the difference between the stress fields measured before and after the 4-point bending test.

(ii) The second one consists of an inversion of the data obtained during the test by using our forward model. 


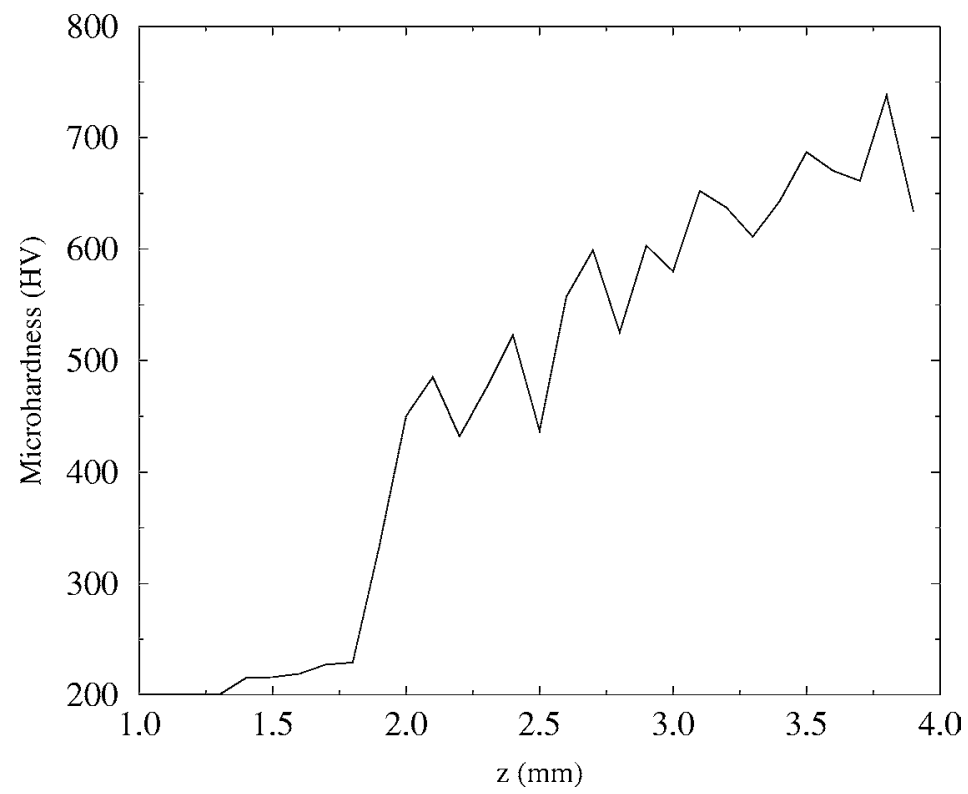

Figure 5. Vickers microhardness as a function of depth.

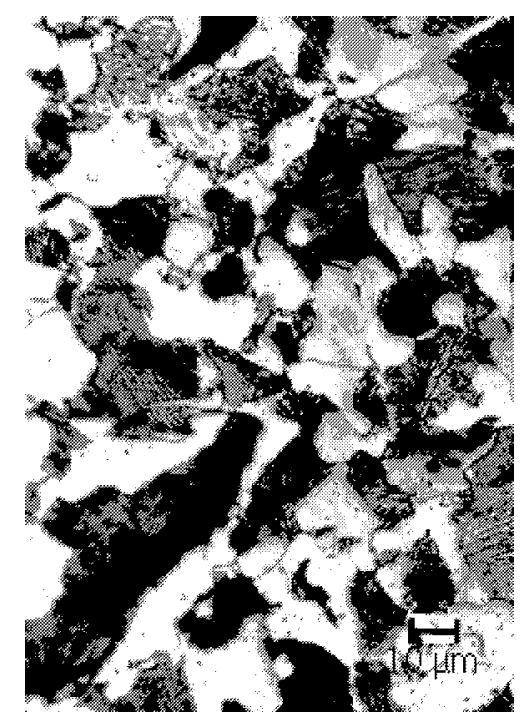

Figure 6. Ferrito-perlitic microstructure.

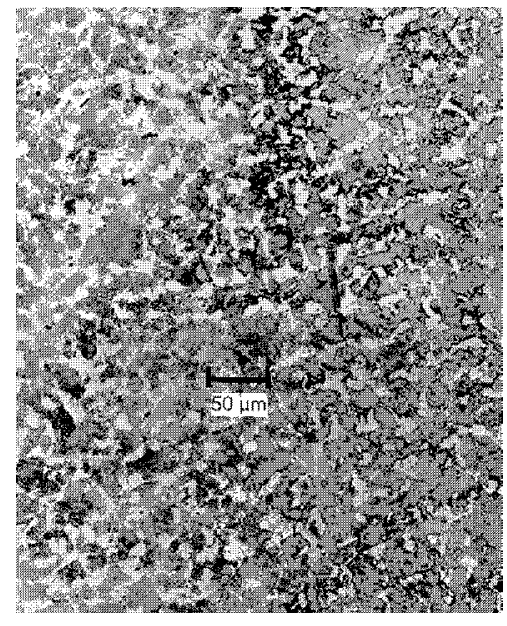

Figure 7. Mixed zone.

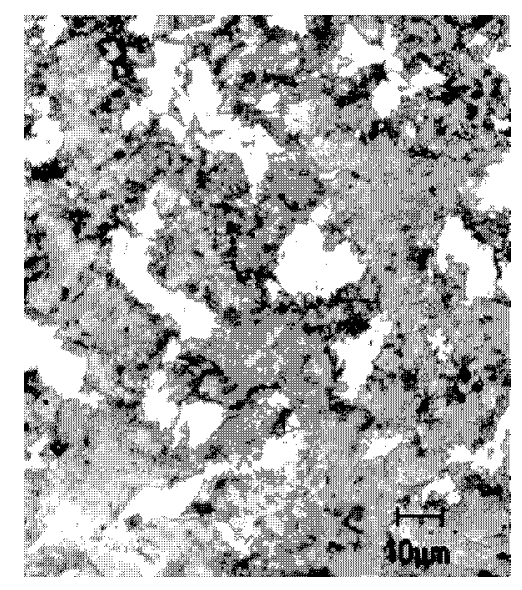

Figure 8. Ferrito-martensitic microstructure.

\subsection{Method based on difference in stress fields}

The stress field $\boldsymbol{\sigma}^{\mathrm{f}}$ which is present in the test bar after the bending test has been conducted, is, according to Hooke's law (7),

$$
\sigma^{\mathrm{f}}=\sigma^{0}+C:\left(\varepsilon^{\mathrm{f}}-\boldsymbol{\varepsilon}^{\mathrm{p}}\right)
$$


in which $\boldsymbol{\varepsilon}^{\mathrm{f}}$ is the final strain field. We observe that if the fields $\boldsymbol{\sigma}^{0}, \boldsymbol{\sigma}^{\mathrm{f}}$ and $\boldsymbol{\varepsilon}^{\mathrm{f}}$ are known, then the final plastic strain can be calculated using the equation above, which can also be written as:

$$
\alpha_{i}=\varepsilon_{i}^{\mathrm{f}}-\frac{\sigma_{i}^{\mathrm{f}}-\sigma_{i}^{0}}{E_{i}} \quad(i=1,2,3) .
$$

Recall that the reference state for total strain and plastic strain is the state of the test bar before bending. The final strain field $\varepsilon^{\mathrm{f}}$ is then calculated thanks to the indications given by the strain gauges located on the two sides $z=h$ and $z=-h$ during the test (see figure 1 ) by solving the system (6).

The measurements of the initial and final stress fields $\sigma_{0}$ and $\sigma^{\mathrm{f}}$ are performed using matter removal and $\mathrm{X}$-ray diffraction. This technique being destructive, the measurement of $\sigma^{0}$ cannot be done before bending. It has to be done on a second test bar, called a reference test bar, which is assumed to be identical to the first in terms of geometry and heat treatment.

Assuming a purely elastic unloading of the 4-point bending test, the final plastic strain field is the same as the plastic strain field induced by maximum loading. Furthermore, the stress field at the maximum loading $\left(M=M_{\mathrm{M}}\right)$ satisfies

$$
\sigma_{1}^{\mathrm{M}}(z)=\sigma_{1}^{\mathrm{f}}(z)-z \frac{M_{\mathrm{M}}}{I}, \quad \sigma_{i}^{\mathrm{M}}=\sigma_{i}^{\mathrm{f}} \quad(i=2,3) .
$$

We have then obtained both stress $\boldsymbol{\sigma}^{\mathrm{M}}$ and plastic strain $\boldsymbol{\varepsilon}^{\mathrm{p}}$ at the maximum loading. In planes corresponding to the coordinate $z$ in which plastic strains are non zero, the yield criterion is satisfied at the maximum loading and provides the relation

$$
\sqrt{Q\left(\sigma^{\mathrm{M}}(z)-H(z) \boldsymbol{\varepsilon}^{\mathrm{p}}(z)\right)}-k(z)=0 .
$$

The identification of $k$ and $H$ is found in the previous equation, and is only possible in planes which have sustained irreversible straining. It is then necessary to impose a large maximum force $F_{\mathrm{M}}$ to yield all the layers which have been affected by the heat treatment. Furthermore, this treatment generates compressive stresses on both sides of the test bar but the bending test generates compressive stresses only on side $(u)$, which ends up being the side on which it is more convenient to conduct the identification process.

For a given coordinate $z$, the only equation of use is (16), in which the two unknown profiles $k(z)$ and $H(z)$ are present. It is then found convenient to assume specific types of variations with depth for them. Among the various possibilities, one consists of dividing the thickness $[-h, h]$ of the test bar into several intervals $\left[z_{i}, z_{i+1}\right]$, on which $k(z)$ and $H(z)$ have a constant value $k_{i}$ and $H_{i}$ respectively. For simplicity sake, the intervals $\left[z_{i}, z_{i+1}\right]$ may correspond to the step adopted during the measurement of the surface stresses. The pair $\left(k_{i}, H_{i}\right)$ is obtained by enforcing (16) at $z=z_{i}$ and $z=z_{i+1}$ and thus solves the system of two equations $(j=i, i+1)$

$$
\frac{3}{2}\left(\left(\boldsymbol{\sigma}^{\mathrm{M}}\right)^{\prime}\left(z_{j}\right):\left(\boldsymbol{\sigma}^{\mathrm{M}}\right)^{\prime}\left(z_{j}\right)\right)-3 H_{i}\left(\left(\boldsymbol{\sigma}^{\mathrm{M}}\right)^{\prime}\left(z_{j}\right): \boldsymbol{\varepsilon}^{\mathrm{p}}\left(z_{j}\right)\right)+\frac{3}{2} H_{i}^{2}\left(\boldsymbol{\varepsilon}^{\mathrm{p}}\left(z_{j}\right): \boldsymbol{\varepsilon}^{\mathrm{p}}\left(z_{j}\right)\right)-k_{i}^{2}=0 .
$$

We point out however that the determination of constants $H_{i}$ using this technique leads to scattered, and even negative, values of $H$. This can be explained by the strong sensitivity of the $H_{i}$ 's to the values of $\boldsymbol{\varepsilon}^{\mathrm{p}}\left(z_{i}\right)$ and $\boldsymbol{\varepsilon}^{\mathrm{p}}\left(z_{i+1}\right)$. Accuracy on field $\boldsymbol{\varepsilon}^{\mathrm{p}}$ is less than the accuracy on field $\boldsymbol{\sigma}^{\mathrm{M}}$, since the estimation of the former needs the measurement of the stresses before bending $\left(\sigma_{0}\right)$, and not only the measurement of the stresses after bending $\left(\sigma^{\mathrm{f}}\right)$.

Another approach is to find $H(z)$ by other means, e.g., using the second method to be presented, then evaluate the field $k(z)$ from (16). 


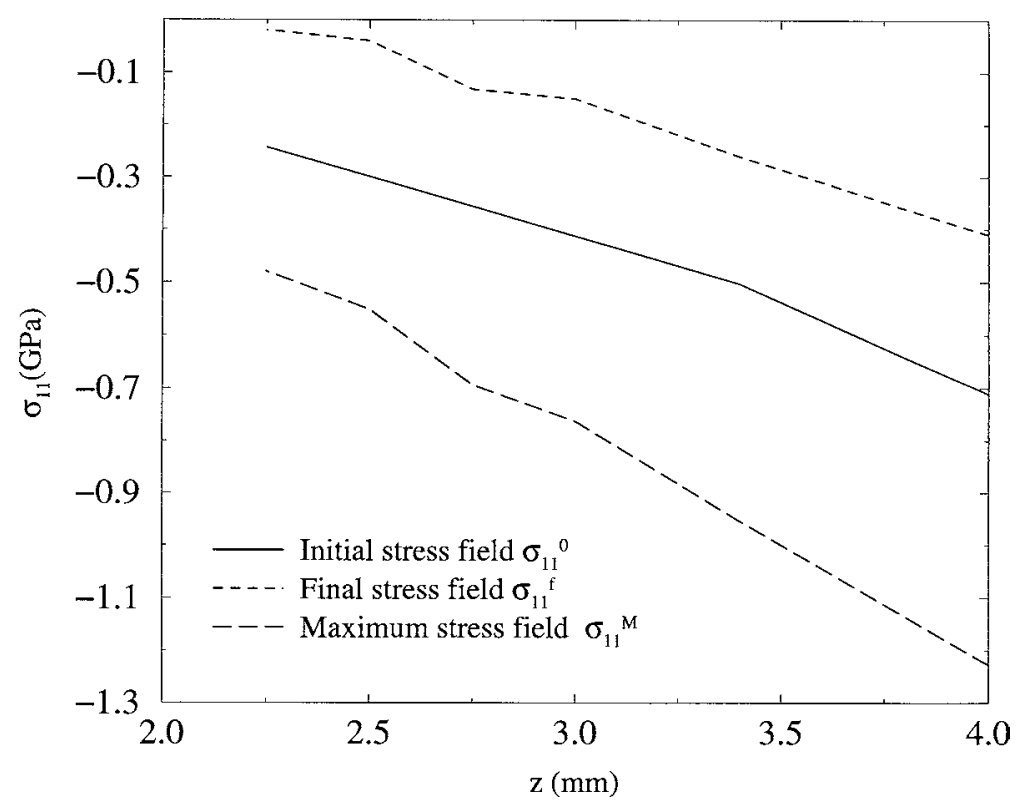

Figure 9. Component $\sigma_{11}$ as a function of depth for initial, final and maximum load conditions.

To show the effect of bending on the stress field, we have presented the components $\sigma_{11}^{0}(z), \sigma_{11}^{\mathrm{f}}(z)$ and $\sigma_{11}^{\mathrm{M}}(z)$ as a function of $z$ in figure 9 , and the components $\sigma_{22}^{0}(z), \sigma_{22}^{\mathrm{f}}(z)=\sigma_{22}^{\mathrm{M}}(z)$ in figure 10, the latter being obtained after post-treatment of a particular 4-point bending test.

In figure 11, we present the results concerning $k(z)$ with given values $H(z)$ obtained using the second method (see further). The two curves correspond to two different test bars, which are supposed to have been submitted to the same heat treatment but which have been bent differently (the maximum bending moments are respectively $M_{\mathrm{M}} \cdot L_{2}=182 \mathrm{~N} \cdot \mathrm{m}$ and $\left.M_{\mathrm{M}} \cdot L_{2}=190 \mathrm{~N} \cdot \mathrm{m}\right)$.

\subsection{Method based on modelling of the bending test}

The second identification method, which is more classical, relies on the modelling of the bending test discussed in Section 3.5.

\subsubsection{Principle}

The aim is to adjust the functions $k(z)$ and $H(z)$ in the affected layer so that the responses of the real and the simulated structures are the same for the same loading history. To apply this method, we first have to define which observations are used for the identification, and, secondly, to specify the search domain for the unknown functions $k(z)$ and $H(z)$.

As far as the first point is concerned, we study the evolution of the curvature $\gamma_{11}=\gamma$ of the test bar, which depends on the applied moment $M_{11}=-M$. The curvature $\gamma$ is measured by the strain gauges thanks to relation (6) and $M$ is provided by relation (5). We only use the increasing part of the loading since the unloading is elastic. More precisely, $n+1$ points $\left(M_{j}, \gamma_{j}\right)$ of the experimental curve $\gamma=f(M)$ are known, with $\left(M_{0}, \gamma_{0}\right)=(0,0)$.

The theoretical curve $\gamma=f(M)$ is obtained by using the algorithm described in Section 3.5, and by applying $n$ loading steps $\Delta M_{j}=M_{j+1}-M_{j}$ from $M=0$, resulting in $n+1$ values of calculated curvature $\gamma_{j c}$ starting 


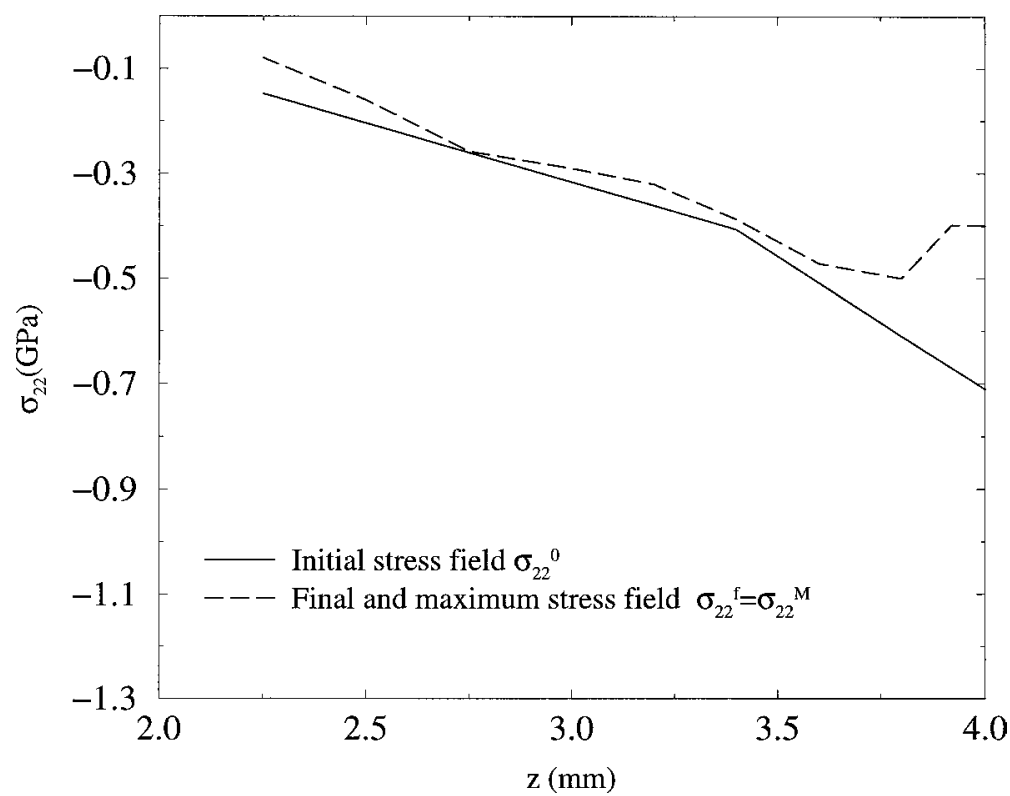

Figure 10. Component $\sigma_{22}$ as a function of depth for initial, final and maximum load conditions.

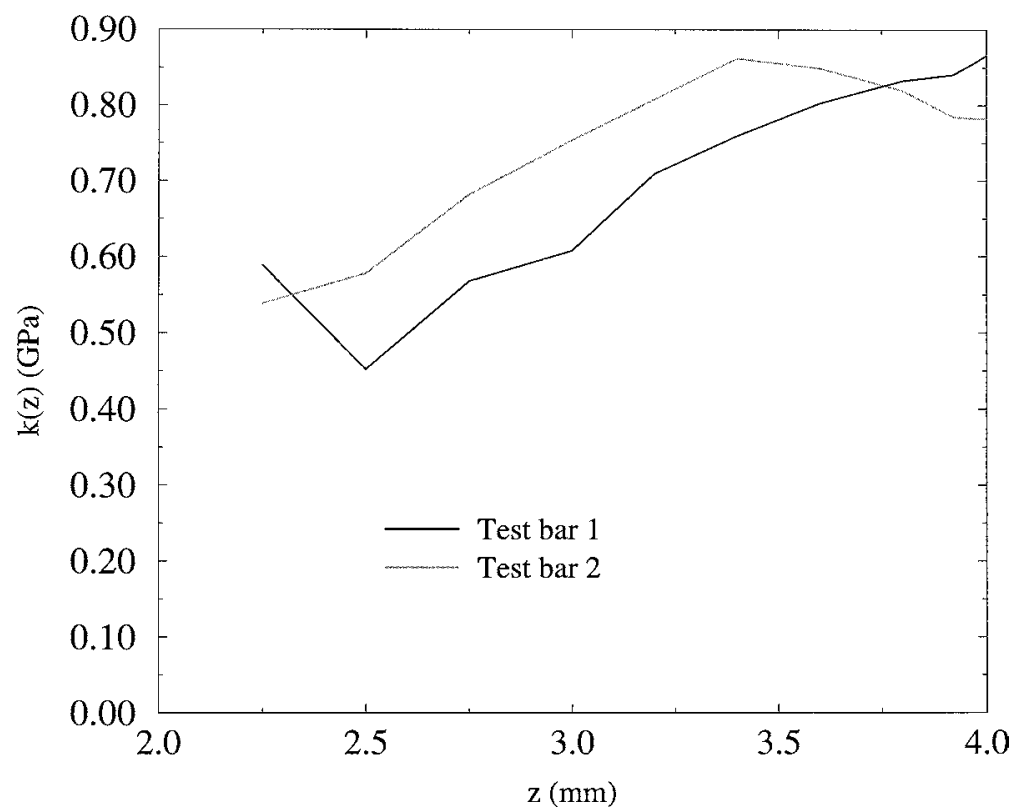

Figure 11. Shape of the tensile yield strength $k(z)$.

with $\gamma_{0}=0$. The curvature $\gamma_{j c}$ being a function of $k$ and $H$, we propose to minimize the cost junction $J$ defined by

$$
J(k, H)=\frac{1}{2} \sum_{j=1}^{n}\left(\gamma_{j c}(k, H)-\gamma_{j}\right)^{2}
$$




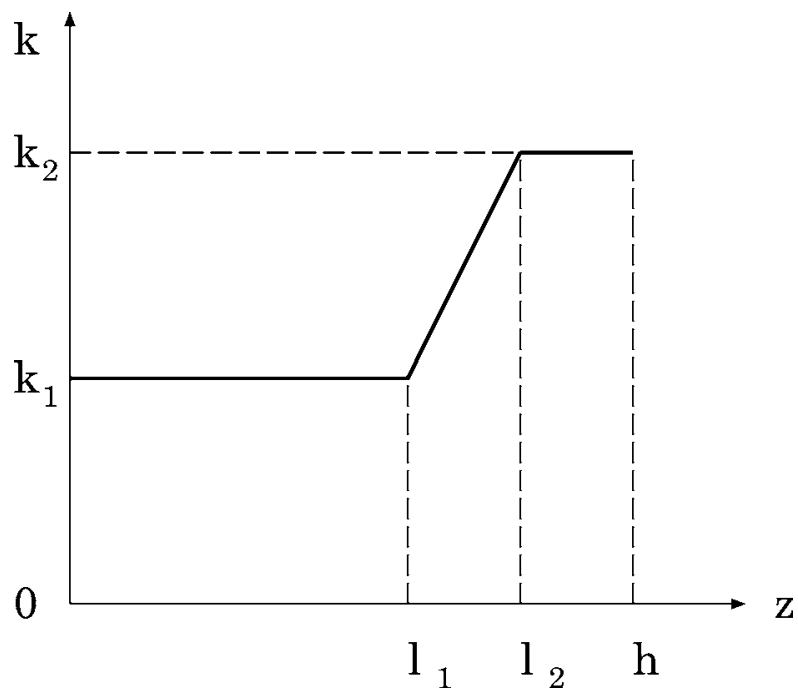

Figure 12. A priori shape of $k(z)$.

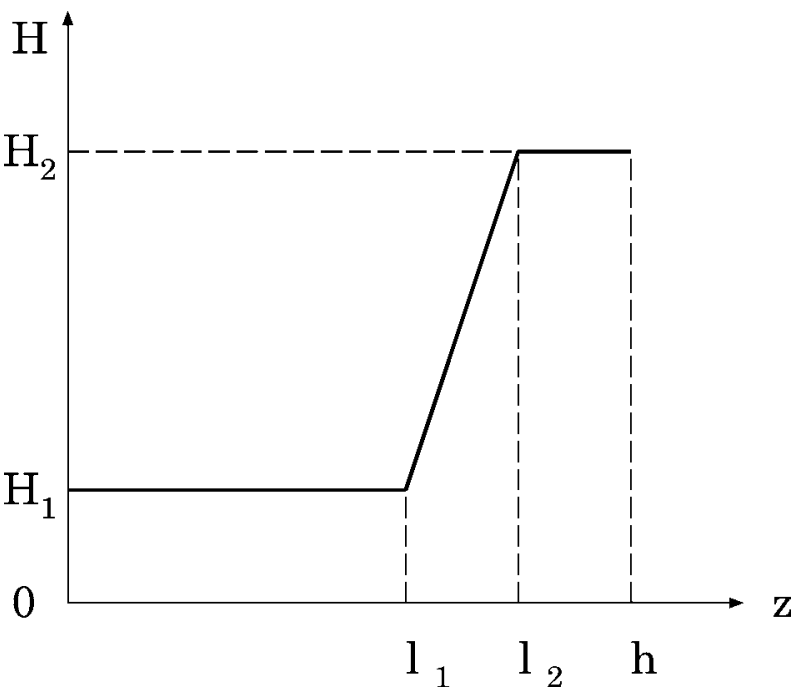

Figure 13. A priori shape of $H(z)$.

in terms of $k$ and $H$.

Concerning the domains in which $k(z)$ and $H(z)$ are searched, it should be mentioned that they have to be sufficiently large so that the experimental curve is correctly reproduced by the forward modelling, but sufficiently small so that this good accuracy does not happen for pairs $(k(z), H(z))$ that are very different from each other. We propose to base our forward modelling on simple variations of $k$ and $H$, deduced from our previous observations on the microstructure (see the end of Section 4), which have revealed that the thickness is divided into three zones corresponding each to a particular microstructure (ferrito-perlitic, mixed and ferritomartensitic) and that microhardness is an increasing function of $z$. The chosen simplified shapes $k(z)$ and $H(z)$, represented in figures 12 and 13 , are defined by parameters $k_{1}, l_{1}, H_{1}, k_{2}, l_{2}$ and $H_{2}$.

To model the response of the test bar, we also have to make use of the stress field $\sigma^{0}$, which is determined from the reference test bar. The components of $\sigma^{0}\left(\sigma_{12}^{0}\right.$ can be neglected with respect to $\sigma_{11}^{0}$ and $\left.\sigma_{22}^{0}\right)$ are reconstructed by matter removal on both sides. Note again that they are obtained up to a certain depth and need to be extended to the whole depth. This extension should ensure that the stress field is statically (equilibrium) and plastically (yield criterion) admissible.

A key assumption of the second method is to assume that the two components $\sigma_{11}^{0}(z)$ and $\sigma_{22}^{0}(z)$ have the same profiles and can be appropriately described by a single function $s_{0}(z)$ defined in terms of the two parameters $\sigma_{h}^{0}$ and $l_{0}$ (see figure 14). This function $s_{0}(z)$ is even and corresponds to a parabola in the interval $\left[0, l_{0}\right]$, taking a zero value at $l_{0}$. This function is then linear on $\left[l_{0}, h\right]$ taking for value $\sigma_{h}^{0}$ at $h$. The function $s_{0}(z)$ is compared to measurements in figure 15 in which the parameters $l_{0}$ and $\sigma_{h}^{0}$ are set to $2.2 \mathrm{~mm}$ and -740 $\mathrm{MPa}$, respectively.

The function $s^{0}$ respects the statical admissibility of the initial stress field $\boldsymbol{\sigma}_{\mathbf{0}}$. This initial stress field must also be plastically admissible, this condition being dependent on the unknown shape $k(z)$.

Before presenting the numerical application, let us summarize the different parameters involved in the modelling of the bending test (forward calculation):

(i) the elastic constants $E$ and $\nu$,

(ii) the profiles of the tensile yield strength $k(z)$ and of the modulus of strain hardening $H(z)$, which are functions of $k_{1}, k_{2}, H_{1}, H_{2}, l_{1}$ and $l_{2}$, 


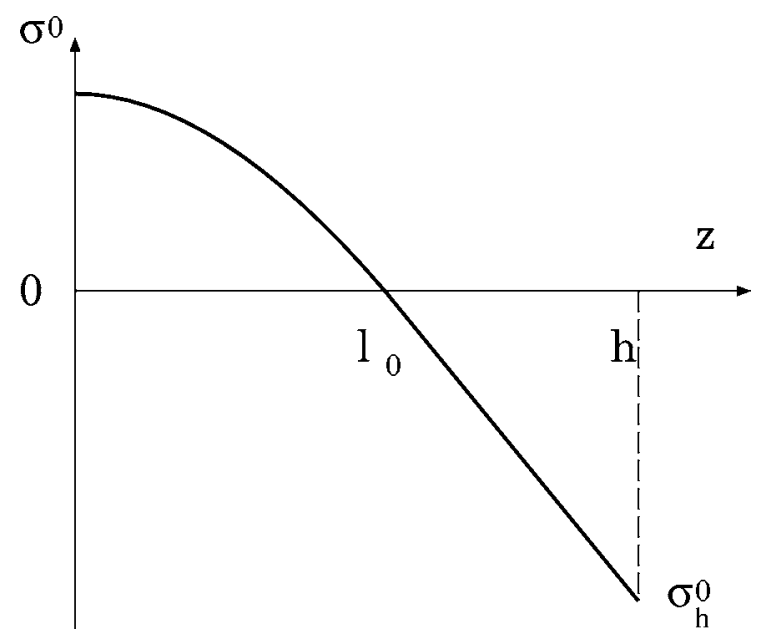

Figure 14. Function $s^{0}$ adopted to model the variation with depth of $\sigma_{11}^{0}$ and $\sigma_{22}^{0}$.

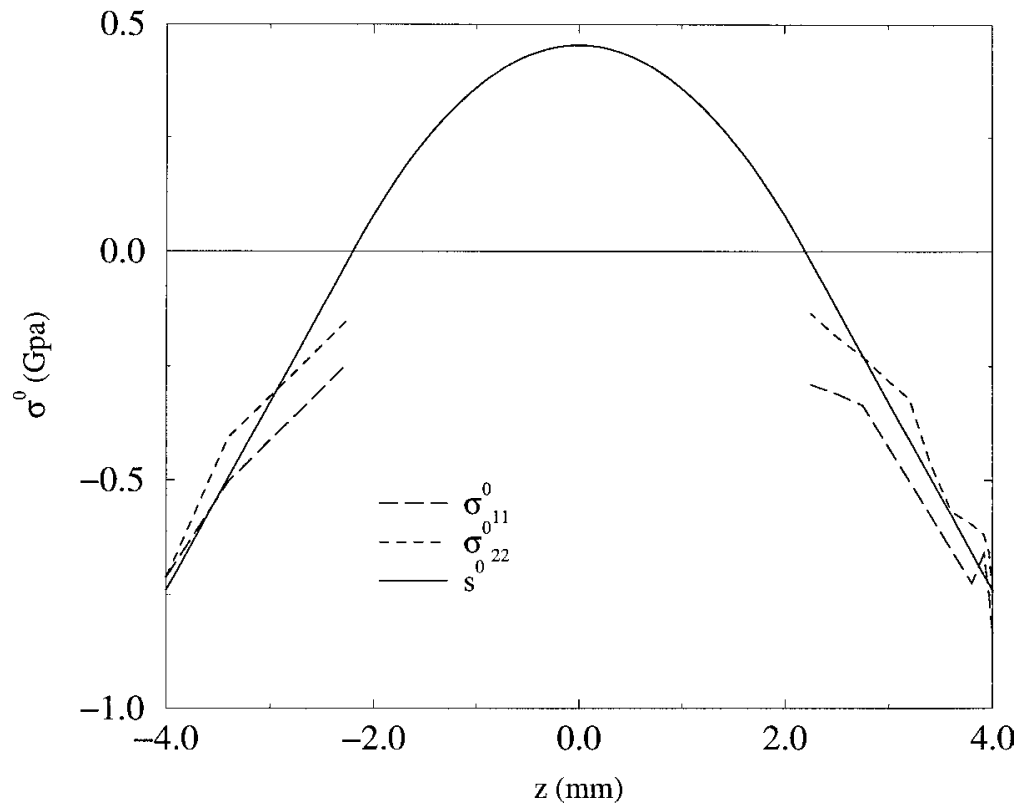

Figure 15. Measured components $\sigma_{11}^{0}$ and $\sigma_{22}^{0}$, and comparison with the function $s_{0}$.

(iii) the initial stress field $\sigma^{0}$, which is a function of $l_{0}$ and $\sigma_{h}^{0}$.

Among these parameters, we consider that $E, v, k_{1}, H_{1}, l_{0}$ and $\sigma_{h}^{0}$ are known. The first four concern the untreated material and are obtained by means of a classical tensile test, the last two are obtained from residual stress measurements on the reference test bar. Thus, the unknown parametres are $k_{2}, H_{2}, l_{1}$ and $l_{2}$.

The cost function $J(17)$ of fields $k(z)$ and $H(z)$ hence reduces to a function $J_{s}$ of vector $\boldsymbol{p}=\left(k_{2}, H_{2}, l_{1}, l_{2}\right)$ :

$$
J_{s}(\boldsymbol{p})=\frac{1}{n} \sqrt{\sum_{j=1}^{n}\left(\frac{\gamma_{j c}(\boldsymbol{p})-\gamma_{j}}{\gamma_{j}}\right)^{2}},
$$


and a minimizer $\hat{\boldsymbol{p}}$ of $J_{s}(\boldsymbol{p})$ is sought.

\subsubsection{Numerical application}

Let us now study the minimization of $J_{s}$. The difficulties are:

(i) the parameters $p_{i}$ have different physical meanings and dimensions,

(ii) the values of $\gamma_{j c}(\boldsymbol{p})(j=1, \ldots, n)$ are obtained by performing a forward elastic-plastic calculation. The elastic-plastic law is not sufficiently smooth to allow an exact calculation of the gradient of the function $J_{s}$ by using an adjoint state.

The first difficulty can be solved by normalizing the problem. We first define a search domain $\left[p_{\min }^{1}, p_{\max }^{1}\right] \times$ $\cdots \times\left[p_{\min }^{N}, p_{\max }^{N}\right]$ of the $N$ parameters, the average values of which are $p_{\text {moy }}^{1}, \ldots, p_{\text {moy }}^{N}$. We transform the parameter $\boldsymbol{p}$ into a dimensionless parameter $\tilde{\boldsymbol{p}}$ by setting $\tilde{\boldsymbol{p}}=D^{-1} \boldsymbol{p}, D$ being the diagonal $N \times N$ matrix formed by the previous average values. A new cost function $\tilde{J}_{s}$ is then defined by setting

$$
\tilde{J}_{S}(\tilde{\boldsymbol{p}})=J_{s}(\boldsymbol{p})
$$

the gradient of $\tilde{J}_{s}$ being

$$
\tilde{J}_{s}^{\prime}(\tilde{\boldsymbol{p}})=D J_{s}^{\prime}(\boldsymbol{p})
$$

For the second difficulty, since we do not know the exact expression of gradient $J_{s}^{\prime}$, we have chosen to approximate it using finite differences.

A subsidiary difficulty is to take some constraints on parameters into account. First, $l_{1}$ and $l_{2}$ satisfy $l_{1} \leqslant l_{2}$. Besides, the field $k(z)$ must be such that the initial stress field $\sigma_{0}$ is plastically admissible, that's to say $\sqrt{Q\left(\sigma_{0}\right)}-k<0$. This condition results in

$$
k_{1} \geqslant-\frac{3}{4} \frac{h-l_{0}}{l_{0}} \sigma_{h}^{0}, \quad k_{2} \geqslant-\sigma_{h}^{0} .
$$

The first inequality concerns known parameters and has already been taken into account through the choice of $\sigma_{h}^{0}$. The second inequality has to be accounted by the optimization. Constraints $l_{1} \leqslant l_{2}$ and $k_{2} \geqslant-\sigma_{h}^{0}$ are imposed by projections in the domain of admissible parameters.

The quasi-Newton algorithm with BFGS's updating formula has been used (see for example (Gill et al., 1982) or (Bonnans et al., 1997)). A numerical application of our procedure is now presented, the control parameters being $k_{2}, H_{2}$, and $l_{2}$. We assume $l_{1}=l_{0}$, which means that the domain initially in compression corresponds to the domain in which the properties of the material have been altered by heat treatment. This assumption relies on the experimental estimations of $l_{0}$, given by the measurement of $\sigma_{0}(z)$, and $l_{1}$, given by the observations of the microstructure at different depths. The two parameters $l_{0}$ and $l_{1}$ are approximately the same. The dimensions of the test bars are $L_{1}=220 \mathrm{~mm}, L_{2}=21 \mathrm{~mm}$ and $h=4 \mathrm{~mm}$, the length between $B$ and $C$ is set to $110 \mathrm{~mm}$ $(d=45 \mathrm{~mm})$. The elastic and plastic constants of the untreated material are $E=200 \mathrm{GPa}, v=0.3, k_{1}=455$ $\mathrm{MPa}$ and $H_{1}=6.5 \mathrm{GPa}$. The two parameters which define the initial stress field, through the function $s_{0}$, are $l_{0}=2.2 \mathrm{~mm}$ and $\sigma_{h}^{0}=-740 \mathrm{MPa}$ (the same as the ones used to draw figure 15).

The identification is based on the two bending tests which have already been exploited for the first method, enabling the results on the tensile yield strength $k(z)$ to be compared. The values of optimal parameters $k_{2}, H_{2}$ and $l_{2}$ which have been obtained for the two bending tests are represented in table $I$.

Figure 16 shows the experimental and computed bending curves (bending moment versus curvature) for optimal parameters $k_{2}, H_{2}$ and $l_{2}$, and for each bending test. Finally, figures 17 and 18 present the optimal 
Table I. Computed values of $k_{2}, H_{2}$ and $l_{2}$ in the second method.

\begin{tabular}{llll}
\hline & $k_{2}(\mathrm{MPa})$ & $H_{2}(\mathrm{GPa})$ & $l_{2}(\mathrm{~mm})$ \\
\hline Test bar 1 & 749 & 60 & 3.88 \\
Test bar 2 & 741 & 53.5 & 3.89 \\
\hline
\end{tabular}

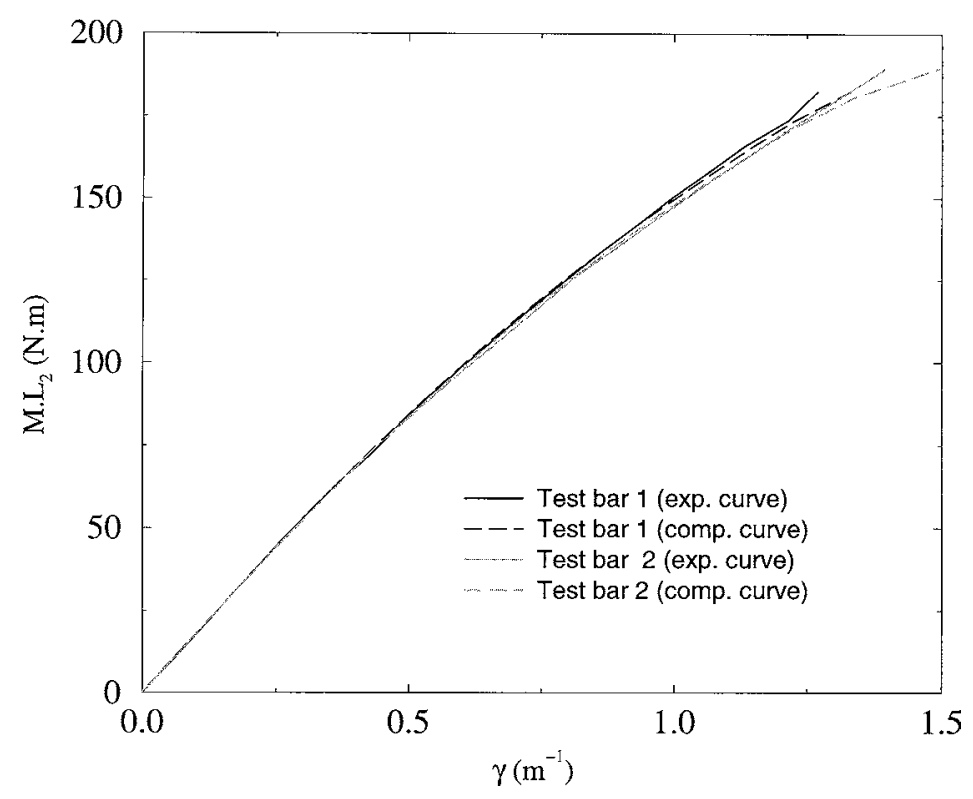

Figure 16. Experimental and computed bending curves.

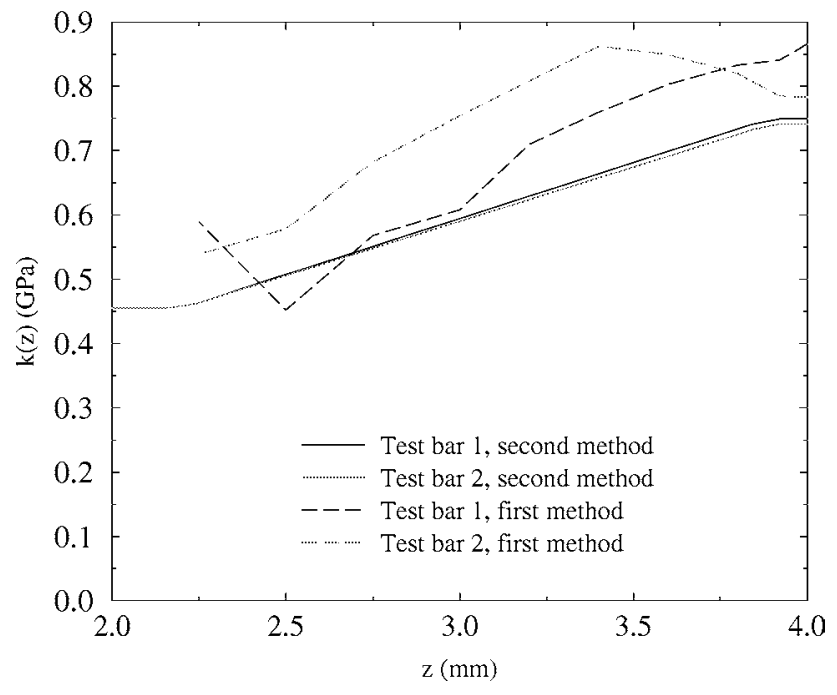

Figure 17. Identified profile $k(z)$.

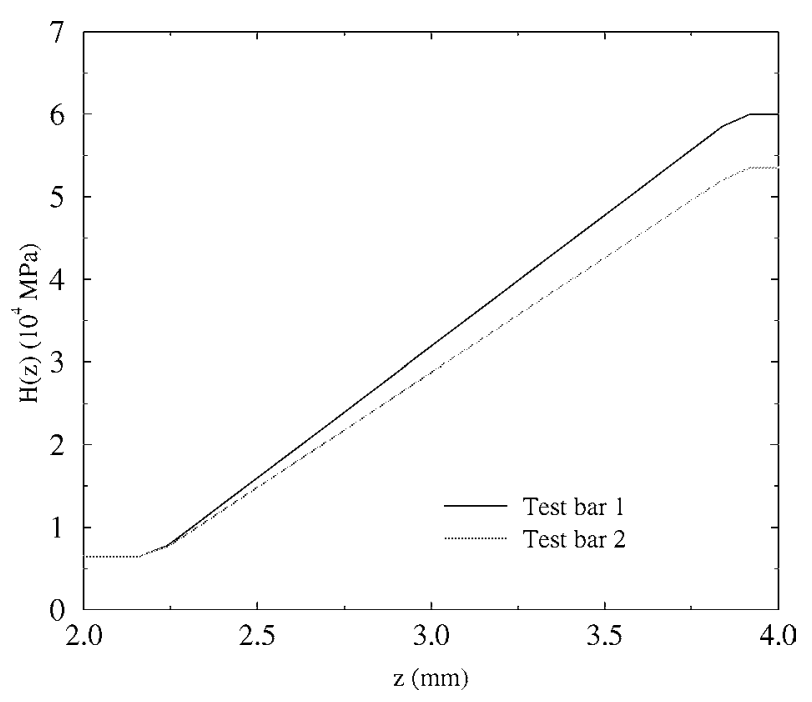

Figure 18. Identified profile $H(z)$. 
shapes $k(z)$ and $H(z)$ corresponding to each bending test, the first shape being compared to the one obtained by the first method.

We note that the optimization of three parameters enables simulation of the bending test with an excellent accuracy. Furthermore, the experimental bending curves are very close to each other, which suggests that the effects of heat treatment are approximately the same for all test bars. Finally, the agreement between the shapes $k(z)$ obtained by the two methods is quite good.

\subsubsection{A sensitivity test}

To complete this study, the influence of the affected thickness, which corresponds to $h-l_{1}=h-l_{0}=h-l$ according to our model, on the accuracy of the identified parameters $k_{2}, H_{2}$ and $l_{2}$, is now considered. The interest of this sensitivity analysis is to determine whether our method is still applicable in the case of superficial treatments leading to smaller thicknesses of the affected layer.

To that purpose, the following numerical test is performed. For given values of $k_{2}, \mathrm{H}_{2}$ and $l_{2}$, we compute the bending test using the algorithm of Section 3.5 for increasing values of $l$ and obtain bending curves defining equation $\gamma=f_{l}(M)$. We add a small perturbation to each curve by replacing the previous equation with the two equations $\gamma=(1 \pm \delta) f_{l}(M), \delta$ being constant and much smaller than 1 . This perturbation corresponds to experimental defects such as imperfectly known kinematical conditions or errors in the model itself. We consider these curves as experimental results, and calculate the optimal parameters $k_{2}^{ \pm}(\delta, l), H_{2}^{ \pm}(\delta, l)$ and $l_{2}^{ \pm}(\delta, l)$ by using our optimization algorithm. The sensitivity of the second method can be quantified by the values of $\Delta k_{2}(\delta, l) / k_{2}$, with

$$
\frac{\Delta k_{2}}{k_{2}}(\delta, l)=\frac{\left|k_{2}^{+}(\delta, l)-k_{2}^{-}(\delta, l)\right|}{k_{2}},
$$

$\Delta H_{2}(\delta, l) / H_{2}$ and $\Delta l_{2}(\delta, l) / l_{2}$.

Numerical calculations are performed with the constant parameters $k_{1}=435 \mathrm{MPa}, H_{1}=6.5 \mathrm{GPa}, \sigma_{h}^{0}=$ $-710 \mathrm{MPa}, k_{2}=750 \mathrm{MPa}, H_{2}=60 \mathrm{GPa}$ and $l_{2}=3.8 \mathrm{~mm}$. The increasing values of $l$ are $2.2 \mathrm{~mm}, 2.55 \mathrm{~mm}$, $29 \mathrm{~mm}, 32.5 \mathrm{~mm}$ and $36 \mathrm{~mm}$. Figures 19,20 and 21 present the values of $\Delta k_{2} / k_{2}, \Delta H_{2} / H_{2}$ and $\Delta l_{2} / l_{2}$ as functions of the proportion of affected material, $(h-l) / l$, for the two different values of $\delta 0.01$ and 0.02 . Figure 22 presents the average cost function $J_{s}$ corresponding to the optimal parameters $k_{2}^{+}, H_{2}^{+}$and $l_{2}^{+}$on the one hand, to the optimal parameters $k_{2}^{-}, H_{2}^{-}$and $l_{2}^{-}$on the other hand, as a function of the proportion of affected material and for the two values of $\delta$. We note from figures 19-21 that the stability of the different parameters (at least for $k_{2}$ and $\mathrm{H}_{2}$ ) decreases as the proportion of the affected material decreases. This is quite logical since this proportion must be sufficiently large so that the influence of the plastic parameters which characterize the affected layer on the bending curve is sufficiently strong. The average cost function corresponding to the optimal parameters increases when the proportion of the affected material decreases, which is due to the projections of the parameters in the domain defined by the constraints $k_{2} \geqslant-\sigma_{h}^{0}$ and $l_{2} \leqslant h$. If the proportion of affected material decreases, $k_{2}^{-}$and $l_{2}^{+}$tends to go out of this domain. Enforcing the previous inequalities increases the difference between the "experimental" and optimal curves.

\section{Conclusions}

In this paper, we have proposed an inverse approach to determine the variation with depth $z$ of the tensile yield strength $k(z)$ and of the strain hardening modulus $H(z)$ within a layer of steel affected by induction hardening, using a bending test on a parallelepipedic bar.

The inverse problem has been solved by two methods, first by measuring the residual stresses after the bending test, second by modelling the bending test. The first method is simple from a theoretical point of view, 


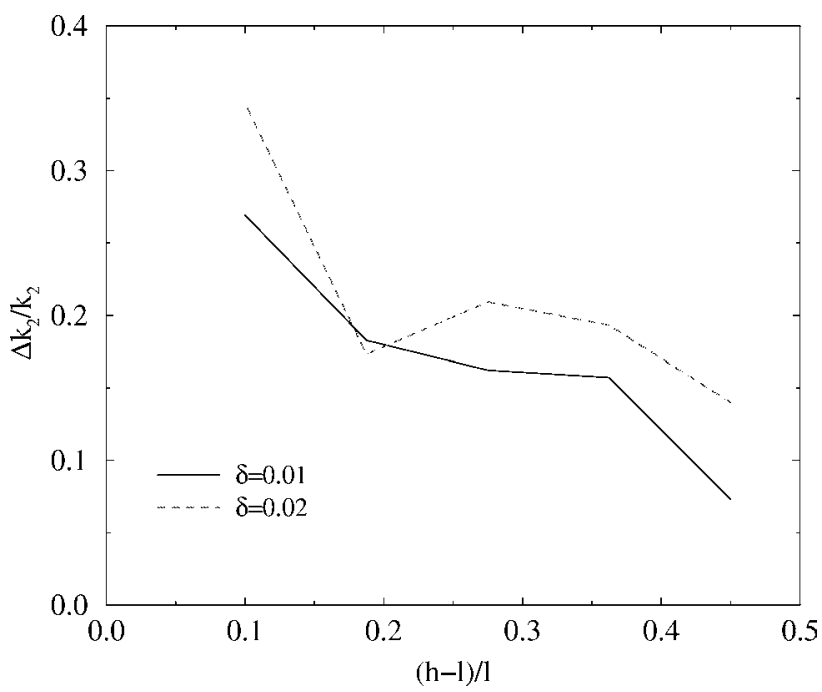

Figure 19. Influence of the proportion of affected material on the sensitivity of $k_{2}$.

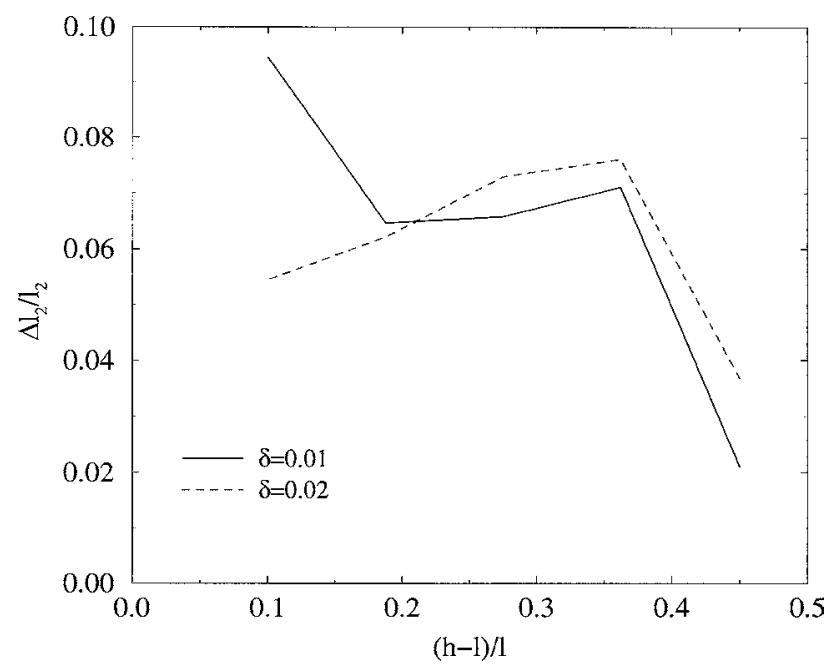

Figure 21. Influence of the proportion of affected material on the sensitivity of $l_{2}$.

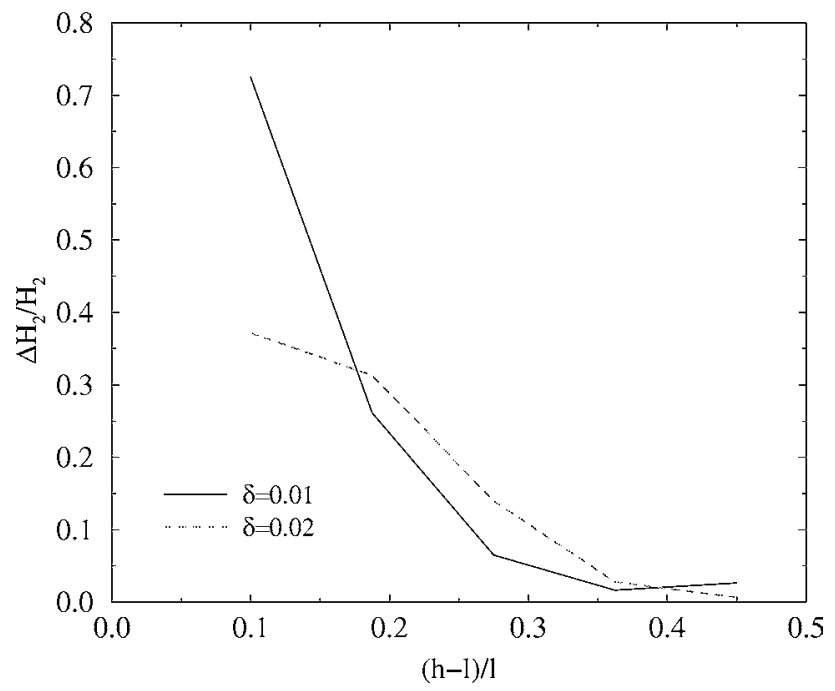

Figure 20. Influence of the proportion of affected material on the sensitivity of $\mathrm{H}_{2}$.

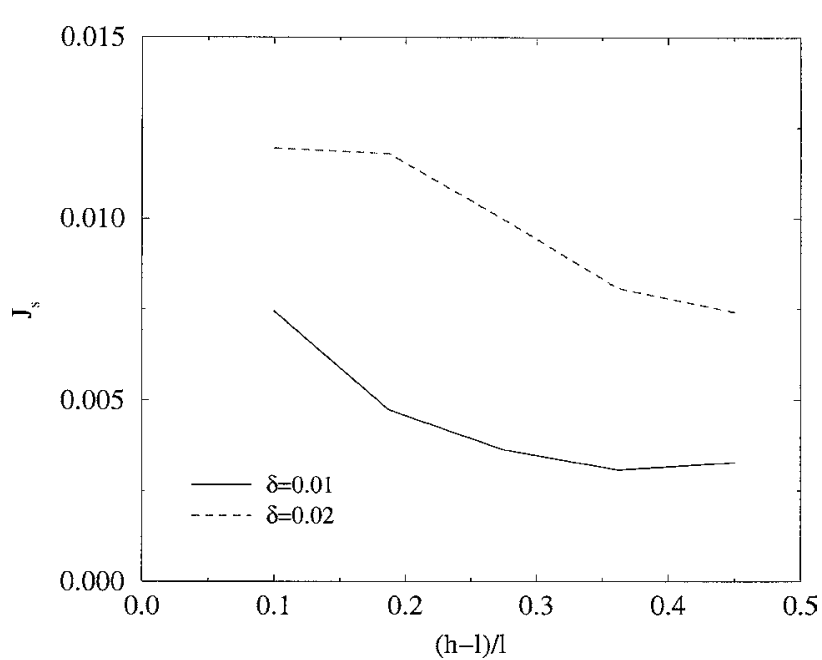

Figure 22. Average cost function for optimal parameters.

but the technique of X-ray diffraction requires a long time of experimental work. The main drawback of this method is that the number of unknowns is too large compared to the number of measurements, whose accuracy are low. As a consequence, the fields of interest, in particular $H(z)$, are not determined with a satisfactory accuracy.

The second method partly solves this problem, by relying on all experimental points of the bending curve and only a few parameters characterizing the general shapes of $k(z)$ and $H(z)$. The main drawback of this method is that these shapes have to be chosen a priori. Microstructural observations have been essential to guide these choices. Furthermore, this method needs the provision of the initial stress field in all the bar, and 
thus to extend the stress field measured in a certain outer layer to the rest of the bar. This extension also implies a priori choices.

Comparing the two methods, it turns out that the second gives better results than the first, especially for the hardening modulus $H(z)$. It must be pointed out that the quality of the identification strongly depends on the accuracy within which the characteristics of the untreated material are known.

Our numerical sensitivity test shows the limitations of the method. The sensitivity of the identified profiles with respect to experimental errors increases as the thickness of the affected layer decreases, so that the identification is only possible if the proportion of affected material is sufficiently large, say at least $20 \%$. Our approach can be adapted to other superficial treatments than induction hardening, such as, flame quenching, LASER quenching, shot-peening and deep rolling. However, our approach would probably be inadequate for techniques such as plasma-coating which generate too thin layers. As far as treatments like nitriding, carbonitriding and case hardening are concerned, our method would necessitate modifications, since such treatments not only alter the plastic properties of the material but also the elastic ones.

\section{Acknowledgements}

We thank M. Bonnet and Y. Leroy for helpful discussions.

\section{References}

Ballard P., Constantinescu A., 1994. On the inversion of subsurface residual stresses from surface stress measurements. J. Mech. Phys. Solids 42, 1767-1787.

Barralis J.P., Maeder G., 1983. Précis de Métallurgie. Collection Précis, AFNOR/Nathan.

Bonnans J.F., Gilbert J.C., Lemaréchal C., Sagastizabál C., 1997. Optimisation Numérique, Springer.

Bourgeois L., 1998. Contrôle Optimal et Problèmes Inverses en Plasticité. Thèse de doctorat, Ecole Polytechnique.

Davies J., Simpson P., 1979. Induction Heating Handbook. Macgraw-Hill.

François D., Pineau A., Zaoui A., 1992. Elasticité et Plasticité. Hermes.

Gill P.E., Murray W., Wright M.H., 1982. Practical Optimization. Academic Press.

Lebrun J.L., 1996. Détermination des contraintes résiduelles par rayons X. Document de formation générale, ENSAM.

Moore M.G., Evans W.P., 1956. Mathematical correction for stress in removed layers in X-Ray diffraction residual stress analysis. Meeting of the SAE Residual Stress Commitee, Pittsburg, Aug. 7-8.

Nguyen Q.S., 1977. On the elastic plastic initial-boundary value problem and its numerical integration. Internat. J. Numer. Methods Engrg. 11, 817-832. 